\title{
GENERATION OF THE LOWER CENTRAL SERIES
}

\author{
by ROBERT M. GURALNICK
}

(Received 12 September, 1980)

0. Introduction. Let $G$ be a group. The $r$ th term $L_{r} G$ of the lower central series of $G$ is the subgroup generated by the $r$-fold commutators

$$
\Gamma_{r} G=\left\{\left[x_{0}, \ldots, x_{r}\right] \mid x_{i} \in G\right\},
$$

where $\left[x_{0}\right]=x_{0},\left[x_{0}, x_{1}\right]=x_{0}^{-1} x_{1}^{-1} x_{0} x_{1}$, and for $r>1$,

$$
\left[x_{0}, \ldots, x_{r}\right]=\left[\left[x_{0}, \ldots, x_{r-1}\right], x_{r}\right] \text {. }
$$

Dark and Newell [1, Theorem 1] proved that if $G$ is nilpotent and $L_{r} G$ is cyclic, then $L_{r} G=\Gamma_{r} G$. In this paper, we generalize this and obtain:

THEOREM A. Suppose $r \geq 2$. There exists a group $G$ with $L_{r} G$ cyclic of order $n$ and $L_{r} G \neq\left(\Gamma_{r} G\right)^{k}$ if and only if $n=p_{1}^{\alpha_{1}} \ldots p_{m}^{\alpha_{m}}$, where the $p_{i}$ are distinct primes and $m \geq 2^{k+1}-1$.

The case $r=1$ has been studied in great detail (see [3], [7] and [8]). For $r=1$, the condition $m \geq 2^{k+1}-1$ must be replaced by a more complicated set of conditions (see [4, Theorem 5]). In section 2, we show that if $L_{r} G=\langle a\rangle$ with $a \in\left(\Gamma_{r} G\right)^{k}$, then $L_{r} G=\left(\Gamma_{r} G\right)^{k+1}$. Some results for $L_{r} G$ a rank 2 abelian p-group are given in section 3 . In particular, an example is constructed to show that for $r \geq 2$, this does not imply $L_{r} G=\Gamma_{r} G$.

1. Proof of Theorem A. We first consider an example. This is a generalization of one of MacDonald [7].

ExAmple 1.1. Denote the nonempty subsets of $\{1, \ldots, k\}$ by $\alpha_{1}, \alpha_{2}, \ldots, \alpha_{s}$, where $s=2^{k}-1$. Let $A_{1}, \ldots, A_{s}$ be nontrivial abelian groups. Then we can choose abelian groups $B_{1}, \ldots, B_{s}$ so that

$$
A_{i}=\left\{b^{2^{r}} \mid b \in B_{i}\right\} \quad(i=1, \ldots, s) .
$$

Let $E=\left\langle x_{1}, \ldots, x_{k}\right\rangle$ be an elementary abelian group of order $2^{k}$. Consider $G=$ $\left(B_{1} \times \ldots \times B_{s}\right) E$ (semidirect), where if $b \in B_{j}$, then

$$
x_{i} b x_{i}=\left\{\begin{array}{lll}
b & \text { if } & i \in \alpha_{j}, \\
b^{-1} & \text { if } & i \notin \alpha_{j} .
\end{array}\right.
$$

It is easily seen that $L_{r} G=A_{1} \times \ldots \times A_{s}$. We claim that if $r \geq 2$ and $1 \neq a_{i} \in A_{i}$ for each $i$, then

$$
\left(a_{1}, \ldots, a_{s}\right) \in L_{r} G-\left(\Gamma_{r} G\right)^{k-1} .
$$

To see this suppose $c \in\left(\Gamma_{2} G\right)^{k-1} \supseteq\left(\Gamma_{r} G\right)^{k-1}$. It is straightforward to verify that this implies

$$
c=\prod_{i=1}^{k-1}\left[\left(b_{i 1}, \ldots, b_{i s}\right), y_{i}\right] \text {, }
$$

Glasgow Math. J. 23 (1982) 15-20. 
where $b_{i j} \in B_{j}$ and $y_{i} \in E$ for $i=1, \ldots, k-1$. Let $H$ be a hyperplane of $E$ containing $\left\langle y_{1}, \ldots, y_{k-1}\right\rangle$. Now each $C_{E}\left(B_{j}\right)$ is a hyperplane and $C_{E}\left(B_{j}\right) \neq C_{E}\left(B_{k}\right)$ unless $j=k$. Thus since there are $s$ hyperplanes, $H=C_{E}\left(B_{i}\right)$ for some $j$. Thus the $j$ th component of $c$ is 1 , proving the claim.

This proves the sufficiency of Theorem $A$ by taking $A_{i}$ to be cyclic of order $p_{i}^{\alpha_{i}}$. For necessity, we need some preliminary results.

LEMMA 1.2. If $A$ is an abelian normal subgroup of $G$, then $\left[A, x_{1}, \ldots, x_{r}\right] \subseteq \Gamma_{r} G$.

Proof. The map sending $a \rightarrow\left[a, x_{1}, \ldots, x_{r}\right]$ is an endomorphism of $A$. Hence its image is $\left[A, x_{1}, \ldots, x_{r}\right]$.

Lemma 1.3. Suppose $G$ is a finite group and $x \in\left(\Gamma_{r} G\right)^{k}$. If $x$ has order $m$ and $(e, m)=1$, then $x^{e} \in\left(\Gamma_{r} G\right)^{k}$.

Proof. Set

$$
\phi(g)=\left|\left\{\left(g_{i j}\right) \mid g=\prod_{i=1}^{k}\left[g_{i o}, \ldots g_{i r}\right]\right\}\right| .
$$

Clearly $g \in\left(\Gamma_{r} G\right)^{k}$ if and only if $\phi(g) \neq 0$. Also $\phi$ is a class function. Hence

$$
\phi=\sum a_{\mathbf{x}} \chi
$$

where the sum runs over the irreducible complex characters of $G$. Gallagher [2, Equation 4] has shown that the $a_{x}$ are rational. Let $\theta$ be a primitive $m$ th root of 1 and $\sigma$ an automorphism with $\sigma(\theta)=\theta^{e}$. Thus

$$
\begin{aligned}
\phi(x) & =\sigma(\phi(x)) \\
& =\sum a_{x}(\sigma \chi(x)) \\
& =\sum a_{x} \chi\left(x^{e}\right) \\
& =\phi\left(x^{e}\right) .
\end{aligned}
$$

In particular, $\phi(x)=0$ if and only if $\phi\left(x^{e}\right)=0$.

LEMMA 1.4. If $L_{r} G$ is finite, then there exist a finite group $H$ and an isomorphism $\varphi$ of $L_{r} G$ and $L_{r} H$ such that $\varphi\left(\Gamma_{s} G\right)=\Gamma_{s} H$ for all $s \geq r$.

Proof. By passing to a subgroup, we can assume $G$ is finitely generated. Now $G$ is nilpotent-by-finite. Hence $G$ has a torsion-free characteristic subgroup $T$ of finite index (cf. [9, p. 153]). Let $H=G / T$. If $s \geq r$, then

$$
L_{s} H=T L_{s} G / T \cong L_{s} G /\left(T \cap L_{s} G\right) \cong L_{s} G \text {. }
$$

Clearly $\Gamma_{s} G$ and $\Gamma_{s} H$ correspond under this isomorphism.

We need one more lemma to obtain the result of Dark and Newell for $L_{r} G$ finite cyclic. 
Lemma 1.5. Let $P$ be a $p$-group with $L_{r} P$ cyclic. Suppose $P=\langle I\rangle$. If $x \in L_{r} P$, there exist $t \in G$ and $t_{1}, \ldots, t_{r} \in I$ such that $\langle x\rangle=\left\langle\left[t, t_{1}, \ldots, t_{r}\right]\right\rangle$.

Proof. Since $P=\langle I\rangle$, there exist $t_{0}, \ldots, t_{r} \in I$ such that $y=\left[t_{0}, \ldots, t_{r}\right]$ is a generator of $L_{r} P$. If $x \in L_{r+1} P$, the result follows by induction since $\left|L_{r+1} P\right|<\left|L_{r} P\right|$. Otherwise

$$
x=y^{e} \equiv\left[t_{0}^{e}, t_{1}, \ldots, t_{r}\right]\left(\bmod L_{r+1} P\right),
$$

and so $\langle x\rangle=\left\langle\left[t_{0}^{e}, t_{1}, \ldots, t_{r}\right]\right\rangle$.

THEOREM 1.6 (Dark-Newell [1]). If $G$ is nilpotent and $L_{r} G$ is a finite cyclic group, then $L_{r} G=\Gamma_{r} G$.

Proof. By Lemma 1.4, we can assume $G$ is finite, and so we can take $G$ a $p$-group. The result now follows from Lemmas 1.3 and 1.5.

Dark and Newell [1] also proved the result for $L_{r} G$ infinite cyclic without assuming $G$ nilpotent. Rodney [8] proved the above results for $r=1$. The assumption that $G$ is nilpotent can be weakened. Set

$$
L_{\infty} G=\bigcap_{i=1}^{\infty} L_{i} G
$$

THEOREM 1.7. If $L_{r} G$ is finite cyclic and $L_{\infty} G$ has order $p^{e}$, then $L_{r} G=\Gamma_{r} G$.

Proof. By Lemma 1.4 and Theorem 1.6, we can assume $G$ is finite and $e \geq 1$. Let $P \in \operatorname{Syl}_{p}(G)$. Since $H=L_{\infty} G \subset P, P$ is normal in $G$ and thus has a complement $T$. Note that $K=L_{r} G \cap P \supset H \neq 1$. As $K$ is cyclic, $L_{r} P \subset K=[T, K]=H$. So if $x \in L_{r} G$, then $x=h u$ with $h \in H$ and $u \in L_{r} T$. Since $T$ is nilpotent, by Lemma 1.5 , there exist $t \in T$ and $t_{1}, \ldots, t_{r} \in T-C_{T}(H)$ so that $\langle u\rangle=\left\langle\left[t, t_{1}, \ldots, t_{r}\right]\right\rangle$. Now since $t_{i} \in T-C_{T}(H)$ and $(|T|, p)=$ $1,\left[H, t_{i}\right]=H$, and so $\left[H, t_{1}, \ldots, t_{r}\right]=H$. Thus there exists $y \in H$ so that $\left[y, t_{1}, \ldots, t_{r}\right]=h$. Hence $\langle h u\rangle=\left\langle\left[t y, t_{1}, \ldots, t_{r}\right]\right\rangle$, and so $x=h u \in \Gamma_{r} G$ by Lemma 1.3 .

One more result is needed for Theorem A. Set

$$
[H, x ; 1]=[H, x] \text { and }[H, x ; n]=[[H, x ; n-1], x] .
$$

If $|H|=p_{1}^{\alpha_{1}} \ldots p_{m}^{\alpha_{m}}$, set $\ell(H)=m$.

LeMmA 1.8. Let $A$ be a group acting on a nontrivial cyclic group $H$ such that $[H, A]=H$. Let $r$ be a positive integer.

(a) There exists $x \in A$ such that $\ell(H /[H, x ; r])<\ell(H) / 2$.

(b) If $\ell(H) \leq 2^{k}-3$, there exists $x \in A$ such that $\ell(H /[H, x ; r]) \leq 2^{k-1}-3$.

Proof. Without loss of generality, we can assume $|H|$ is squarefree, and so $[H, x ; r]=$ $[H, x]$ and $H=[H, x] \oplus C_{H}(x)$. Choose $x \in A$ with $\ell([H, x])$ maximal. By induction, there exists $y \in A$ with $\ell([C, y])>\ell(C) / 2$, where $C=C_{H}(x)$. Hence,

$$
\begin{aligned}
\ell([H, x]) & \geq \ell([H, x y]) \\
& \geq \ell([H, x])-\ell([H, x, y])+\ell([C, y]) \\
& =\ell([H, x])-\ell([H, y])+2 \ell([C, y]) .
\end{aligned}
$$


This yields

$$
\ell(C)<2 \ell([C, y]) \leq \ell([H, y]) \leq \ell([H, x])
$$

and proves (a).

To prove (b), it suffices to assume that $\ell(H)=2^{k}-3$ and $k \geq 2$. Choose $x$ and $y$ as above. By (a), $\ell([H, x]) \geq 2^{k-1}-1$. If $\ell([H, x])=2^{k-1}-1$, then $\ell(C)=2^{k-1}-2$, and so $\ell([C, y]) \geq 2^{k-2}$. Then $(*)$ implies that

$$
\ell([H, x]) \geq 2 \ell([C, y]) \geq 2^{k-1},
$$

and the result follows.

Proof of Theorem A. Suppose $L_{r} G$ is cyclic of order $n=p_{1}^{\alpha_{1}} \ldots p_{m}^{\alpha_{m}}\left(m<2^{k+1}-1\right)$. Set $H=L_{\infty} G$. First consider the case $H=L_{r} G$. By Lemma 1.8(a), there exists $x \in G$ with

$$
\ell(H /[H, x ; r])<m / 2 \leq 2^{k}-1 .
$$

By induction and Lemma 1.1,

$$
L_{r} G=\left(\Gamma_{r} G\right)^{k-1}[H, x ; r] \subseteq\left(\Gamma_{r} G\right)^{k}
$$

as desired.

Now assume $H \neq L_{r} G$. Since $H$ is a summand of $L_{r} G$, this implies $\ell(H) \leq 2^{k+1}-3$. We now show that if $\ell(H) \leq 2^{k+1}-3$, then $\left(L_{r} G\right)=\left(\Gamma_{r} G\right)^{k}$. This follows from Theorem 1.7 for $k=1$. If $k \geq 2$, by Lemma $1.8\left(\right.$ b), there exists $x \in G$ with $\ell(H /[H, x ; r]) \leq 2^{k}-3$. As above, we obtain $L_{r} G=\left(\Gamma_{r} G\right)^{k}$.

2. Generators of $L_{r} G$. If $L_{r} G=\langle a\rangle$ and $a \in \Gamma_{r} G$, then all generators of $L_{r} G$ are in $\Gamma_{r} G$ by Lemma 1.3. However, this does not imply $L_{r} G=\Gamma_{r} G$. (See [3] for examples with $r=1$.) Similar examples can be constructed for $r>1$. However, we do obtain:

THeOREM 2.1. If $L_{r} G=\langle a\rangle$ and $a \in\left(\Gamma_{r} G\right)^{e}$, then $L_{r} G=\left(\Gamma_{r} G\right)^{e+1}$.

Proof. If $L_{r} G$ is infinite, then $L_{r} G=\Gamma_{r} G$ by [1, Theorem 4]. If $r=1$, the result follows by [4, Theorem 1]. Thus we can assume $G$ is finite and $r \geq 2$. Let $H=L_{\infty} G$. Thus

$$
a=\prod_{i=1}^{e}\left[t_{0 i}, \ldots, t_{r i}\right]
$$

It follows easily since $r \geq 2$ that

$$
H=\prod_{i=1}^{e}\left[H, t_{r i}\right]=\prod_{i=1}^{e}\left[H, t_{i} ; r\right] \subseteq\left(\Gamma_{r} G\right)^{e} .
$$

Hence by Theorem 1.6, we have $L_{r} G=\left(\Gamma_{r} G\right) H \subseteq\left(\Gamma_{r} G\right)^{e+1}$.

If $r=1$ and $e \geq 2$, then in fact $G^{\prime}=\left(\Gamma_{1} G\right)^{e}$ (see [4, Theorem 1]). We do not know if this is true for $r>1$.

3. Rank 2 Subgroups. By Example 1.1, if $A$ is a finitely generated abelian group with $A=A_{1} \times A_{2} \times A_{3}$, there exists $G$ with $L_{r} G=A$ and $L_{r} G \neq \Gamma_{r} G$. This leaves open the 
case where $L_{r} G$ is a rank $2 p$-group. Dark and Newell [1, Theorem 2] proved in this case that if also $L_{r+1} G=\{1\}$, then $L_{r} G=\Gamma_{r} G$. (See [6] for the case $r=1$.) The author [5, Theorem A] has shown that if $P \in \operatorname{Syl}_{p}\left(G^{\prime}\right)$ is a rank 2 abelian $p$-group, then $P \subseteq \Gamma_{1} G$. We give an example with $p=2$ and $r=2$ with $L_{r} G \neq \Gamma_{r} G$.

Example 3.1. Let $G=\langle x, y, a, b\rangle$ with relations

$$
\begin{gathered}
{[x, y]=b, \quad b^{x}=b^{-1}, \quad b^{y}=b a,} \\
b^{8}=a^{2}=[x, a]=[y, a]=[b, a]=1 .
\end{gathered}
$$

Now

$$
\Gamma_{2} G \subset\left[G^{\prime}, x\right] \cup\left[G^{\prime}, y\right] \cup\left[G^{\prime}, x y\right]=\left\langle b^{2}\right\rangle \cup\langle a\rangle \cup\left\langle a b^{2}\right\rangle .
$$

Hence $a b^{4} \in L_{2} G$, but $a b^{4} \notin \Gamma_{2} G$. Similar examples can be constructed for any $r \geq 2$.

Certain assumptions do guarantee that $L_{r} G=\Gamma_{r} G$.

THEOREM 3.2. Suppose $L_{r} G$ is a rank 2 abelian p-group. If any of the following hold, then $L_{r} G=\Gamma_{r} G$.

(i) $r=1$.

(ii) $L_{r+1} G=\{1\}$.

(iii) $G$ is not nilpotent.

(iv) $L_{r} G$ has exponent $p$.

Proof. As we remarked above, (i) and (ii) are known. We sketch the proof of (iii). So assume $H=L_{\infty} G \neq\{1\}$. As usual, we take $G$ finite. Let $T$ be a complement of $P \in \operatorname{Syl}_{p}(G)$. We can choose $t \in T$ with $[H, t ; r]=[H, t]=H$ (see [5, Lemma 2.6]). Then

$$
L_{r} G=\left\{\left[t s_{0}, \ldots, t s_{r}\right] \mid s_{i} \in P\right\} \subseteq \Gamma_{r} G .
$$

This follows by Theorem 1.6 and induction on $G /[Z P, t]$. Further, (iv) follows since if (ii) and (iii) do not hold, then $L_{r+1} G$ is cyclic and central. Hence $L_{r+1} G \subseteq \Gamma_{r} G$ and if $x, y \in L_{r} G-L_{r+1} G$, then $\langle x\rangle$ and $\langle y\rangle$ are conjugate. Hence $L_{r} G \subseteq \Gamma_{r} G$ by Lemma 1.3.

We close with a conjecture.

CONJECTURE 3.3. There exists a finite set of primes $\Omega$ (perhaps depending on $r$ ) such that if $p \notin \Omega$ and $L_{r} G$ is a rank 2 abelian $p$-group, then $L_{r} G \doteq \Gamma_{r} G$.

For $r=1$ and $L_{1} G=G^{\prime}$ a rank 3 abelian $p$-group, we can take $\Omega=\{2,3\}$ (see [5, Theorem B]).

\section{REFERENCES}

1. R. S. Dark and M. L. Newell, On conditions for commutators to form a subgroup, J. London Math. Soc. (2) 17 (1978), 251-262. (Reviewed in Zbl. Math. 388 (1979), 102).

2. P. X. Gallagher, The generation of the lower central seriés, Canad. J. Math. 17 (1965), 405-410. 
3. B. Gordon, R. Guralnick, and M. Miller, On cyclic commutator subgroups, Aequationes Math. 17 (1978), 241-249

4. R. Guralnick, On cyclic commutator subgroups, Aequationes Math., 21 (1980), 33-38.

5. R. Guralnick, Commutators and commutator subgroups, Advances in Math. (to appear).

6. H. Liebeck, A test for commutators, Glasgow Math. J. 17 (1976), 31-36.

7. I. D. MacDonald, On cyclic commutator subgroups, J. London Math. Soc. 38 (1963), 419-422.

8. D. M. Rodney, On cyclic derived subgroups, J. London Math. Soc. (2) 8 (1974), 642-646.

9. W. R. Scott, Group theory (Prentice-Hall, 1964).

UNIVERSITY OF SOUTHERN CALIFORNIA

Los ANgeles, California 90007 